\title{
TWILLS ON A GIVEN NUMBER OF HARNESSES
}

\author{
W. D. HOSKINS and ANNE PENFOLD STREET
}

(Received 22 December 1980)

Communicated by W. D. Wallis

\begin{abstract}
The simple twills on $n$ harnesses can be classified according to the number of breaks that they possess. An algorithm is detailed for determining these twills and some sample listings given. A formula is derived which evaluates the total number of $n$-harness twills with a specified number of breaks, and hence also the total possible number of twills on $n$ harnesses. Also the balanced twills on $n$ harnesses are enumerated.
\end{abstract}

1980 Mathematics subject classification (Amer. Math. Soc.): 05 B 30, 05 B 45, 62 K 99.

\section{Introduction}

It has been established that the weaving of twill fabrics dates back to at least 1500-1000 BC [2] and the number of different simple twills on $n$ harnesses is well known for at least $n=2,3,4,5,6,7,8$ (Oelsner [8], Laughlin [7], Grünbaum and Shephard [5]). However for $n>8$ no enumeration of the possible structures appears to have been given although a classification has been described in [5] which is closely related to the work of Gilbert and Riordan [4] on symmetry types of periodic sequences; the former is an explicit formula (Theorem 2) for the number of simple twills of period $n$, and the latter gives a recursion relation which may be used to calculate this number. The values were listed in [5] for $n \leqslant 8$ and in [4] for $n \leqslant 20$ (Table $1 ; D_{n} \times \Im_{2}$ ). Before proceeding further it is necessary to introduce the following definitions and describe the environment in which the problem arises.

The second author thanks the Department of Computer Science, University of Manitoba, for hospitality while this work was in progress.

c Copyright Australian Mathematical Society 1982 
The warp on a loom passes through a set of $n$ harnesses ( $n$ an integer $\geqslant 2$ ); when a given harness is raised, some subset of these threads (warp ends) is also raised. It is usual for every warp end to be threaded so that it is associated with a specific harness, although there are historic techniques for ends to be associated with more than one harness (double threading, see [6]). In weaving a particular structure, several harnesses may be lifted simultaneously; so the set of possible lifts constitutes a precise analogue of the positive sets of set theory. Attention will subsequently be restricted to structures in which the shuttle carrying the weft makes a complete uninterrupted motion from one side of the warp to the other. Thus no finger-manipulated weaves are considered; also since the presence of regularity in the woven structure is considered desirable, it is necessary to require periodicity of some type in the interlacements of the warp ends with a single weft pick. It is not necessary to require that the interlacements of successive weft picks with a specified warp end generate the same crossing sequence as the warp ends with the corresponding weft pick; however it does give the simplest possible structure periodic with equal periods along both warp and weft axes, and is generally taken to mean that the pattern has been "tromp as writ" or "woven as drawn in". The following terms can now be conveniently introduced. A binary sequence

$$
S=\left\{s_{i}\right\}
$$

of period $n$ is a sequence of zeros and ones such that

$$
s_{k}=s_{i} \quad(k \equiv i(\bmod n)) .
$$

It may be noted that, if $n \mid m$, then $S$ also has period $m$.

Two binary sequences $S$ and $T$ of period $n$ will be considered equivalent if and only if one can be transformed into the other by a shift, a reversal, complementation, or any finite sequence of these operations. In other words, $S$ and $T$ are equivalent if and only if:

$$
s_{i}=t_{i+1}, \quad s_{i}=t_{n+1-i}, \quad s_{i}=\sim t_{i},
$$

or $S$ can be obtained from $T$ by some finite combination of the above operations.

This means that, if $S$ and $T$ are considered as cyclic sequences of length $n$, then they are equivalent precisely when one can be transformed into the other under the action of the group $D_{2 n} \times \widetilde{S}_{2}$, that is, the direct product of the dihedral group of order $2 n$ with the symmetric group of degree 2 . This is the necklace equivalence discussed by Fine [3] and Gilbert and Riordan [4].

Given a sequence $S=\left\{s_{0}, \ldots, s_{n-1}\right\}$ and two operators $I$ and $P$ such that

$$
S I=S \text { and } S P=\left\{s_{n-1}, s_{n-2}, \ldots, s_{0}\right\},
$$

together with a word

$$
A=I^{j_{1}} P^{j_{2}} I^{j_{3}} P^{j_{4}} \cdots I^{j_{2 r-1}} P^{j_{2 r}},
$$


the sequence $T=\left\{t_{h}\right\}$ of period $m=n \Sigma_{i=1}^{2 r} j_{i}-2 r$ is given by

$$
t_{h}=s_{\lambda} \text { for } h=0,1, \ldots, m-1 \text {, }
$$

where $\lambda$ is defined as follows:

for $n\left(j_{1}+\cdots+j_{i}\right)-i \leqslant h \leqslant n\left(j_{1}+\cdots+j_{i+1}\right)-(i+1)$, we have

$$
\lambda \equiv\left\{\begin{array}{l}
n+1-(h+i), \quad i \text { odd }, \\
h+i, \quad i \text { even. }
\end{array}\right.
$$

Thus the sequence $T$ consists of $j_{1}$ copies of $S$ followed by $j_{2}$ copies of $S P$, and so on, except that where the symbol $s_{0}$ or $s_{n-1}$ is immediately repeated one copy of the symbol is deleted.

EXAMPLE. If

$$
S=00101 \text { (length 5) and } A=I P^{3} \text {, }
$$

then

$$
T=001010100101001010(\text { period } 18)
$$

An interlacement sequence $Q$ of period $n$ is a sequence of over and under crossings of orthogonal strands, and each interlacement sequence can be associated with an equivalence class of binary sequences of the same period.

Definition. A simple twill on $n$ harnesses is a planar interlacement array in which each row (column) of the array is an interlacement sequence of period $n$ and is obtained from the previous row (column) by displacement through one position.

Thus the interlacements of a simple twill can be regarded as a binary array generated as a square tiling by a circulant binary array of period $n$.

EXAmPLE 1. Simple twill on 6 harnesses.

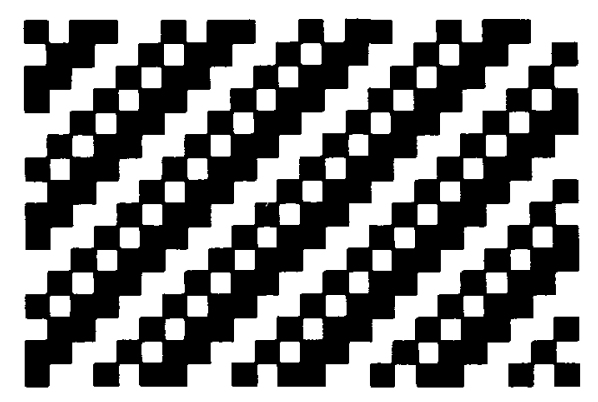


Definition. A point twill on $n$ harnesses is a planar interlacement array in which each row of the array is an interlacement sequence of period $n$. In addition, the array has the property that a row $R$ can be selected such that all subsequent rows can be obtained as follows.

(i) Each of the $n-1$ rows immediately below $R$ is obtained by shifting the previous row to the right, through one position; this set of shifts will be denoted by $R I$.

(ii) Each of the $n-1$ rows immediately below these is obtained by shifting the previous row to the left one position. This set of shifts will be denoted by $(R I) P$. This sequence of sets of shifts then repeats, that is, we have $((((R I) P) I) P) \ldots$ The array above $R$ is a reflection in $R$ of that below.

Notice that in this case, since the operators $I$ and $P$ alternate, at each step the operator acts on the previous row of the array.

EXAMPLe 2. Point twill on 5 harnesses

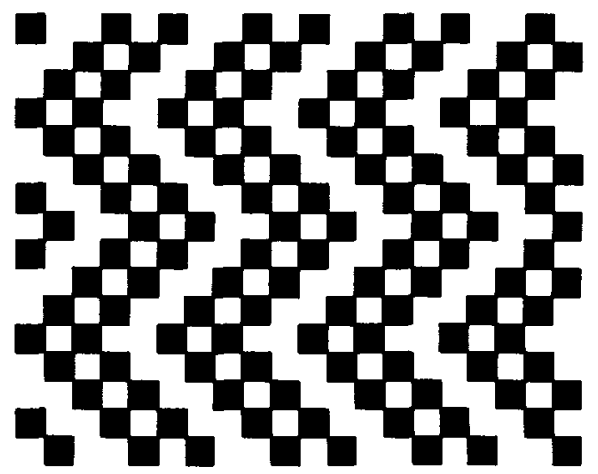

DEFINITION. An extended point twill on $n$ harnesses is a planar interlacement array in which each row of the array is an interlacement sequence of period $n$. In addition, the array has the property that a row $R$ can be selected such that all subsequent rows can be obtained by applying the operators $I$ and $P$, but now arbitrary sequences of $I$ and $P$ are allowed instead of strict alternation.

Thus the interlacement structure $F$ can be precisely described by

$$
F=R A A \ldots
$$

where $R$ is the first row and the word

$$
A=I^{j_{1}} P^{j_{2}} I^{j_{3}} P^{j_{4}} \cdots I^{j_{2 r-1}} P^{j_{2 r}}
$$

lists the operators sequentially applied to $R$. Where two successive operators in the word are different, then the last row obtained from the first of these operators 
becomes the argument to the second of them (just as for the point twill). When two successive operators are the same, then the argument of the first of these operators becomes the argument of the second. This precisely corresponds to the action of the operators $I$ and $P$ on a binary sequence.

Note that, because of the necklace equivalence of periodic sequences, the word $A$ can be considered to represent an equivalence of words which determine equivalent extended point twills. For convenience the representative word $A$, chosen from the equivalence class of words, is that which comes first in the alphabetic ordering.

With the above definition, it becomes apparent that an extended point twill tromp as writ has the same word describing both warp and weft sequences. In particular, the simple twill (see example 1) is an extended point twill where the word

$$
A=I
$$

defines both warp and weft sequences, whereas the point twill (illustrated by example 2) is an extended point twill not tromp as writ where the word

$$
A_{1}=I P
$$

describes the weft (warp) sequences and the word

$$
A_{2}=I
$$

describes the warp (weft) sequences.

EXAMPLE 3. Extended point twill on 5 harnesses not tromp as writ

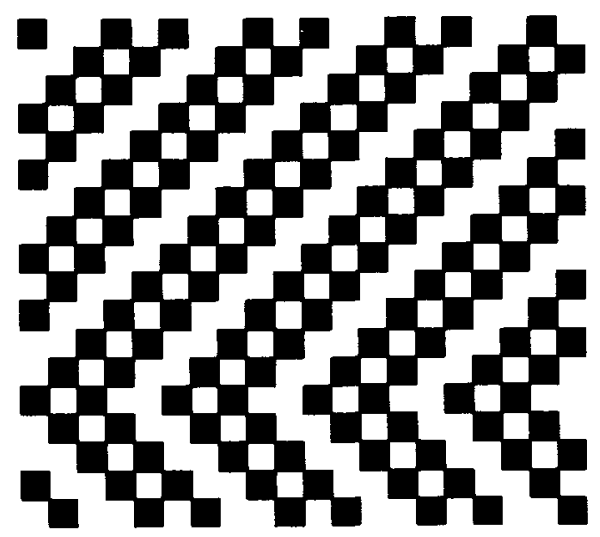


EXAMPLE 4. Extended point twill on 5 harnesses tromp as writ

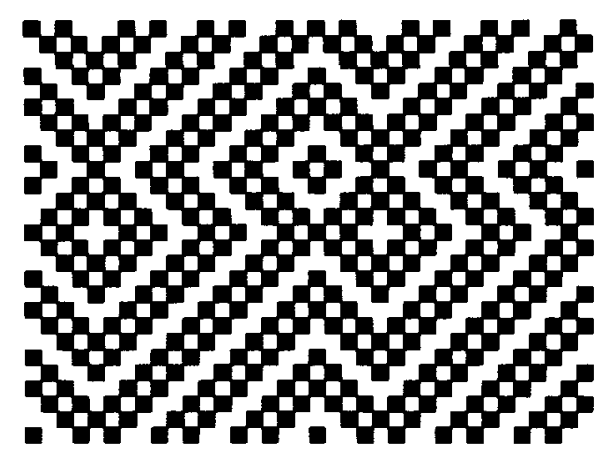

The extended point twill of example 3 has

$$
A_{1}=I P P P \quad \text { and } \quad A_{2}=I
$$

whilst example 4 has

$$
A_{1}=A_{2}=I P P P \text {. }
$$

This means that, to be consistent with the definition of equivalence of sequences, two woven fabrics are regarded as equivalent if one can be transformed into the other by: turning the fabric over; shifting some warp (weft) threads from one side to the other (preserving their cyclic order and interlacements); taking a mirror image of the fabric parallel to either warp or weft directions; interchanging warp and weft; any finite combination of these operations.

Along any strand of a simple twill there are a certain number of breaks per period, that is, a certain number of positions where the strand changes from passing over to passing under the strands at right angles to it, or vice versa.

Algorithm. The simplest method of determining the simple twills on $n$ harnesses is by means of a sieve performed as follows.

(i) A vector $X$ of bits with $2^{n}-2$ components is initialised to ones.

(ii) The index of the first non-zero entry in the vector gives a new twill.

(iii) If $S$ is the $n$-place binary integer form for the integer index in (ii), then all addresses in $X$ corresponding to the decimal integer equivalents of $S$ or of any sequence derivable from $S$ under the action of the direct product $D_{2 n} \times \Xi_{2}$ are set to zero.

(iv) Steps (ii) and (iii) are repeated until done.

In practice, it is obvious that the last twill that can be found for even $n$ is tabby, represented by the sequence $(0101 \ldots 01)$; this is the twill with the maximal number of breaks. For $n$ odd, the last sequence found will be $(001010 \ldots 101)$, 
again with a maximal number of breaks. These two sequences correspond to the integer $\left(4^{[n / 2]}-1\right) / 3$, and this can be used to decrease the dimension of the vector $X$ used in the sieve.

There are considerable economies which may be obtained when this algorithm is implemented in machine code.

EXAmple. To determine the twills on four harnesses, the vector $X$ is

$$
X=\left(\begin{array}{rrrrrrrrrrrrrr}
1 & 2 & 3 & 4 & 5 & 6 & 7 & 8 & 9 & 10 & 11 & 12 & 13 & 14 \\
1 & 1 & 1 & 1 & 1 & 1 & 1 & 1 & 1 & 1 & 1 & 1 & 1 & 1
\end{array}\right)
$$

The index of the first non-zero value is 1; as a 4-place binary integer, it can be written as 0001 .

The cyclic rotations of this give the decimal indices $2,4,8$; the cyclic rotations of the complement give $14,13,11,7$; the other possible operations give no new indices. Therefore, the vector $X$ becomes

$$
X=\left(\begin{array}{rrrrrrrrrrrrrr}
1 & 2 & 3 & 4 & 5 & 6 & 7 & 8 & 9 & 10 & 11 & 12 & 13 & 14 \\
1 & 0 & 1 & 0 & 1 & 1 & 0 & 0 & 1 & 1 & 0 & 1 & 0 & 0
\end{array}\right)
$$

and the next non-zero bit has a decimal index of 3 or, as a binary 4-place, 0011 . The cyclic rotations of this give the decimal indices $6,12,9$, and the cyclic rotations of the complement and other operations yield no new values. So the vector $X$ becomes

$$
X=\left(\begin{array}{rrrrrrrrrrrrrr}
1 & 2 & 3 & 4 & 5 & 6 & 7 & 8 & 9 & 10 & 11 & 12 & 13 & 14 \\
1 & 0 & 1 & 0 & 1 & 0 & 0 & 0 & 0 & 1 & 0 & 0 & 0 & 0
\end{array}\right)
$$

The next non-zero bit has decimal index of 5 (0101 in binary), and this has as a cyclic rotation the value 10 .

Therefore the final vector $X$ is

$$
X=\left(\begin{array}{llllllllllllll}
1 & 0 & 1 & 0 & 1 & 0 & 0 & 0 & 0 & 0 & 0 & 0 & 0 & 0
\end{array}\right)
$$

and indicates there are 3 twills on 4 harnesses corresponding to the decimal integers 1, 3, 5, written as 4-place binary integers. This is a convenient abbreviated method of listing twills that is used for Table 1 to give all possible twills on 12 harnesses. Conversion to standard form is simply accomplished by taking any entry in the table, for example, 5, writing it as a 12-place binary integer 1010000 000000 , and then counting successive runs of ones and zeros, that is,

$$
5 \equiv \frac{11}{19}
$$

in the traditional break notation of weaving. 
TABLE 1

Twills on 12 harnesses in decimal form

$\begin{array}{rrrrrrrrrrr}1 & 3 & 7 & 15 & 31 & 63 & 5 & 9 & 11 & 17 & 19 \\ 23 & 27 & 33 & 35 & 39 & 47 & 51 & 55 & 65 & 67 & 71 \\ 79 & 95 & 99 & 103 & 111 & 119 & 135 & 143 & 195 & 199 & 207 \\ 231 & 455 & 21 & 37 & 43 & 45 & 69 & 73 & 75 & 77 & 83 \\ 87 & 91 & 93 & 107 & 133 & 137 & 139 & 141 & 147 & 151 & 153 \\ 155 & 157 & 163 & 167 & 175 & 179 & 183 & 187 & 189 & 203 & 215 \\ 219 & 273 & 275 & 279 & 283 & 291 & 295 & 307 & 311 & 313 & 315 \\ 327 & 355 & 359 & 371 & 403 & 411 & 423 & 819 & 85 & 149 & 165 \\ 171 & 173 & 277 & 293 & 297 & 299 & 301 & 309 & 325 & 331 & 333 \\ 339 & 343 & 347 & 349 & 363 & 365 & 427 & 585 & 587 & 595 & 603 \\ 619 & 691 & 715 & 717 & 341 & 597 & 661 & 683 & 685 & 693 & 1365\end{array}$

It follows from the definition of necklace equivalence of periodic binary sequences and from the definition of a simple twill that the number of inequivalent simple twills that can be woven on $n$ harnesses is just one less than the number of inequivalent binary sequences of period $n$, for each distinct sequence leads to a distinct twill except for the sequence $000 \ldots$ (or $111 \ldots$ ). It is also of interest to determine the number of simple twills with a given number of breaks per period or, in other words, the number of inequivalent necklaces with a given number of colour changes between successive beads. The number of breaks or colour changes must always be even and will be denoted by $2 k$.

For example, the sequence $\left\{a_{i}\right\}$ of period five

$$
a_{0} a_{1} a_{2} a_{3} a_{4}=11000
$$

has two breaks, one after $a_{1}$ and one after $a_{4}$, and the sequence $\left\{\alpha_{1}\right\}$ of period five

$$
\alpha_{0} \alpha_{1} \alpha_{2} \alpha_{3} \alpha_{4}=10101
$$

has four breaks, after $\alpha_{0}, \alpha_{1}, \alpha_{2}$ and $\alpha_{3}$ respectively.

Each binary sequence $\left\{\alpha_{i}\right\}$ of period $n$ has a unique associated binary sequence $\left\{b_{i}\right\}$, also of period $n$, defined by

$$
b_{i}=a_{i}+a_{i+1} \quad(\bmod 2),
$$

where addition of subscripts is taken modulo $n$. Thus any binary sequence $\left\{a_{i}\right\}$ of period $n$ with $2 k$ breaks has a unique associated binary sequence $\left\{b_{i}\right\}$ containing exactly $2 k$ ones, and any binary sequence $\left\{b_{i}\right\}$ containing exactly $2 k$ ones is the associated sequence of precisely two binary sequences, each with $2 k$ breaks, defined by

$$
a_{i+1, j}=a_{i j}+b_{i j} \quad(\bmod 2), \quad \text { for } j=0,1
$$

where $a_{0,0}=0$ and $a_{0,1}=1$. Hence $\left\{a_{i 0}\right\}=\left\{\overline{a_{i 1}}\right\}$. 
Thus, in the example above, $\left\{a_{i}\right\}=11000$ has the associated sequence $\left\{b_{i}\right\}=$ 01001 , and $\left\{b_{i}\right\}$ is the associated sequence of $\left\{a_{i 0}\right\}=00111$ and its complement $\left\{a_{i 1}\right\}=\left\{a_{i}\right\}$. Similarly, $\left\{\alpha_{i}\right\}$ has the associated sequence $\left\{\beta_{i}\right\}=11110$, which is the associated sequence of $\left\{\alpha_{i 0}\right\}=00001$ and $\left\{\alpha_{i 1}\right\}=\left\{\alpha_{i}\right\}$.

Since both a sequence and its complement have the same associated sequence, the number of equivalence classes of binary sequences of period $n$ with $2 k$ breaks under the action of $D_{2 n} \times \Im_{2}$ is precisely the number of equivalence classes of binary sequences of period $n$ with $2 k$ ones under the action of $D_{2 n}$. This number is most conveniently calculated using Burnside's Lemma.

LemMa (Burnside [1, page 191]). Let $G$ be a finite group, of order $g$, of transformations acting on a finite set $S$, and let two elements of $S$ be equivalent if and only if one can be transformed into the other by a transformation in $G$. Then the number $T$ of inequivalent elements is

$$
T=\frac{1}{g} \sum_{t \in G} I(t),
$$

where $I(t)$ is the number of elements of $S$ left invariant by transformation $t \in G$, and the sum is over all $g$ transformations in $G$.

THEOREM 1. The number $T(n, 2 k)$ of equivalence classes of binary sequences of period $n$ containing precisely $2 k$ ones is given by

$$
T(n, 2 k)=\frac{1}{2 n}\left\{\sum_{d \in D} \Phi(n / d)\left(\begin{array}{c}
d \\
2 k d / n
\end{array}\right)+n\left(\begin{array}{c}
\lfloor n / 2\rfloor \\
k
\end{array}\right)\right\},
$$

where $\Phi$ denotes Euler's totient function and $D$ is the set of positive integers

$$
D=\{d: d|n, n| 2 k d\} \text {. }
$$

Proof. The proof is an application of Burnside's Lemma to the set $S$ of binary sequences of period $n$ containing $2 k$ ones, where the group $G$ is $D_{2 n}$, the dihedral group of order $g=2 n$.

(i) All $\left(\begin{array}{c}n \\ 2 k\end{array}\right)$ of the sequences are left invariant by the identity of $D_{2 n}$.

(ii) Next consider rotation of the cyclic sequence

$$
a_{0} a_{1} \ldots a_{n-1}
$$

through $s$ places, where $d=\operatorname{gcd}(n, s)$. A sequence left invariant by such a rotation must be of the form

$$
a_{0} a_{1} \ldots a_{d-1} a_{0} a_{1} \ldots a_{d-1} \ldots a_{0} a_{1} \ldots a_{d-1},
$$

that is, it must consist of $n / d$ repetitions of a subsequence of length $d$. Since the whole sequence contains $2 k$ ones, the subsequence $a_{0} a_{1} \ldots a_{d-1}$ contains $2 k /(n / d)=2 k d / n$ ones. Thus we need only consider values of $s$ for which $d$ is a 
proper divisor of $n$ such that $n \mid 2 k d$. The number of subsequences of length $d$ containing the required number of ones is $\left(\begin{array}{c}d d \\ k d / n\end{array}\right)$, and any such subsequence determines the whole sequence. Altogether, there are $\Phi(n / d)$ choices of $h$ such that $s=h d$ and $d=\operatorname{gcd}(n, s)$.

Hence, for each $d$ such that $d \mid n, 1 \leqslant d<n$, and $n \mid 2 k d$, there are $\Phi(n / d)$ rotations each leaving $(2 \mathrm{~d} d / n)$ sequences in $S$ invariant.

(iii) Finally, consider the $n$ possible reflections of the cyclic sequence

$$
a_{0} a_{1} \ldots a_{n-1} \text {. }
$$

If $n$ is odd, then each axis of reflection passes through one element of the sequence, say $a_{j}$, and between two others, namely, $a_{j+(n-1) / 2}$ and $a_{j+(n+1) / 2}$. Since the reflection leaves the sequence invariant and since the sequence has $2 k$ ones, we must have $a_{j}=0$; also each of the $(n-1) / 2$ terms on either side of the axis of reflection must include $k$ ones. The number of subsequences of this form is $\left(\begin{array}{c}(n-1) / 2 \\ k\end{array}\right)=\left(\begin{array}{c}\lfloor n / 2\rfloor \\ k\end{array}\right)$, and each of them determines the whole sequence.

If $n$ is even, we have two types of axes of reflection: $n / 2$ axes passing between terms $a_{j}$ and $a_{j+1}$ and again between $a_{j+(n / 2)}$ and $a_{j+1+(n / 2)} ; n / 2$ axes passing through symbols $a_{j}$ and $a_{j+(n / 2)}$. In the first case, the $n / 2$ symbols $a_{j+1}$, $a_{j+2}, \ldots, a_{j+(n / 2)}$ may be chosen in $\left(\begin{array}{c}n / 2 \\ k\end{array}\right)$ ways, so as to include $k$ ones; this determines the sequence. In the second case, $a_{j}=a_{j+(n / 2)}$ and two possibilities arise: either $a_{j}=1$ and the $(n / 2)-1$ symbols $a_{j+1}, a_{j+2}, \ldots, a_{j+(n / 2)-1}$ may be chosen in $\left(\begin{array}{c}(n / 2)-1 \\ k-1\end{array}\right)$ ways to contain $(k-1)$ ones; or $a_{j}=0$ and the $(n / 2)-1$ symbols $a_{j+1}, a_{j+2}, \ldots, a_{j+(n / 2)-1}$ may be chosen in $(\underset{k}{(n / 2)-1})$ ways to contain $k$ ones. In either case this choice determines the whole sequence, so it can arise in $\left(\begin{array}{c}(n / 2)-1 \\ k\end{array}\right)+\left(\begin{array}{c}(n / 2)-1 \\ k-1\end{array}\right)=\left(\begin{array}{c}n / 2 \\ k\end{array}\right)$ ways.

(iv) The total number of invariant sequences is thus

$$
\begin{aligned}
& \left(\begin{array}{c}
n \\
2 k
\end{array}\right) \text { from case (i); } \\
& \sum_{d} \Phi(n / d)\left(\begin{array}{c}
d \\
2 k d / n
\end{array}\right)
\end{aligned}
$$

for $d|n, 1 \leqslant d<n, n| 2 k d$, from case (ii);

$$
n\left(\begin{array}{c}
\lfloor n / 2\rfloor \\
k
\end{array}\right) \text { from case (iii). }
$$

Adding terms from cases (i) and (ii) gives

$$
\sum_{\substack{d|n \\
n| 2 k d}} \Phi(n / d)\left(\begin{array}{c}
d \\
2 k d / n
\end{array}\right)
$$

since $g=2 n$, the Theorem follows. 
COROLlARY 1. The number of n-harness twills with $2 k$ breaks is $T(n, 2 k)$.

COROllary 2. Let $n=2^{h} m$, where $h \geqslant 0$ and $m$ is odd. Then the number of $n$-harness twills is $T(n)$, where

$$
1+T(n) \quad 2^{\left\lfloor 2^{h-1} m-1\right\rfloor}+\frac{1}{2^{h+2} m} \sum_{\lambda=0}^{h} \sum_{\delta \mid m} \Phi(m / \delta) 2^{2^{h-\lambda} \delta+\lambda} .
$$

Proof. Any twill has at least two breaks, or in other words we disregard the trivial case, corresponding to the constant sequence $000 \ldots$ (or equivalently $111 \ldots)$. Hence

$$
\begin{aligned}
1+T(n) & =\sum_{k=0}^{\lfloor n / 2\rfloor} T(n, 2 k) \\
& =\frac{1}{2} \sum_{k=0}^{\lfloor n / 2\rfloor}\left(\begin{array}{c}
\lfloor n / 2\rfloor \\
k
\end{array}\right)+\frac{1}{2 n} \sum_{k=0}^{\lfloor n / 2\rfloor} \sum_{d \in D} \Phi(n / d)\left(\begin{array}{c}
d \\
2 k d / n
\end{array}\right) \\
& =2^{\left\lfloor 2^{k-1} m-1\right\rfloor}+\frac{1}{2 n} \sum_{k=0}^{\lfloor n / 2\rfloor} \sum_{d \in D} \Phi(n / d)\left(\begin{array}{c}
d \\
2 k d / n
\end{array}\right) .
\end{aligned}
$$

By grouping the divisors of $n$ into $h+1$ sets of the form $2^{\lambda} \delta$, where $\delta \mid m$ and $\lambda=0,1, \ldots, h$, and then rearranging summands, we obtain the Corollary.

EXAMPLE. (i) If $n=16=2^{4} .1$, then $h=4, m=1$, and $1+T(16)=2^{7}$ $+\frac{1}{2^{6}}\left(2^{16}+2^{9}+2^{6}+2^{5}+2^{5}\right)=1162$.

(ii) If $n=15=2^{0} .15$, then $h=0, m=15$, and $1+T(15)=2^{6}+$ $\left(1 / 2^{2} .15\right)\left(\Phi(15) \cdot 2+\Phi(5) \cdot 2^{3}+\Phi(3) \cdot 2^{5}+\Phi(1) \cdot 2^{15}\right)=612$.

Table 2 gives the values of $T(n, 2 k)$ and $T(n)$, for $n \leqslant 30$, computed both from the Theorem and Corollary 2 , and by the sieving algorithm of Section 2 .

A twill is said to be balanced if each strand passes over and under those perpendicular to it equally often. Such fabrics are of interest because they do not curl. Each such twill corresponds to a necklace-equivalence class of sequences of (necessarily) even length consisting of equal numbers of zeros and ones. Such sequences will also be called balanced.

THEOREM 2. The number of distinct balanced twills on $n=2 k$ harnesses is

$$
B(2 k)=\frac{1}{8 k}\left\{\sum_{\substack{d \mid 2 k \\
d=2 e}} \Phi(k / e)\left(\begin{array}{c}
2 e \\
e
\end{array}\right)+\sum_{d \mid k} \Phi(2 k / d) 2^{d}+2 k\left(\begin{array}{c}
2\lfloor k / 2\rfloor \\
\lfloor k / 2\rfloor
\end{array}\right)+k .2^{k}\right\} \text {. }
$$

Proof. Burnside's Lemma is applied to the set $S$ of balanced binary sequences of period $2 k$, where the group $G$ is $D_{2.2 k} \times \subseteq_{2}$, the direct product of the dihedral 


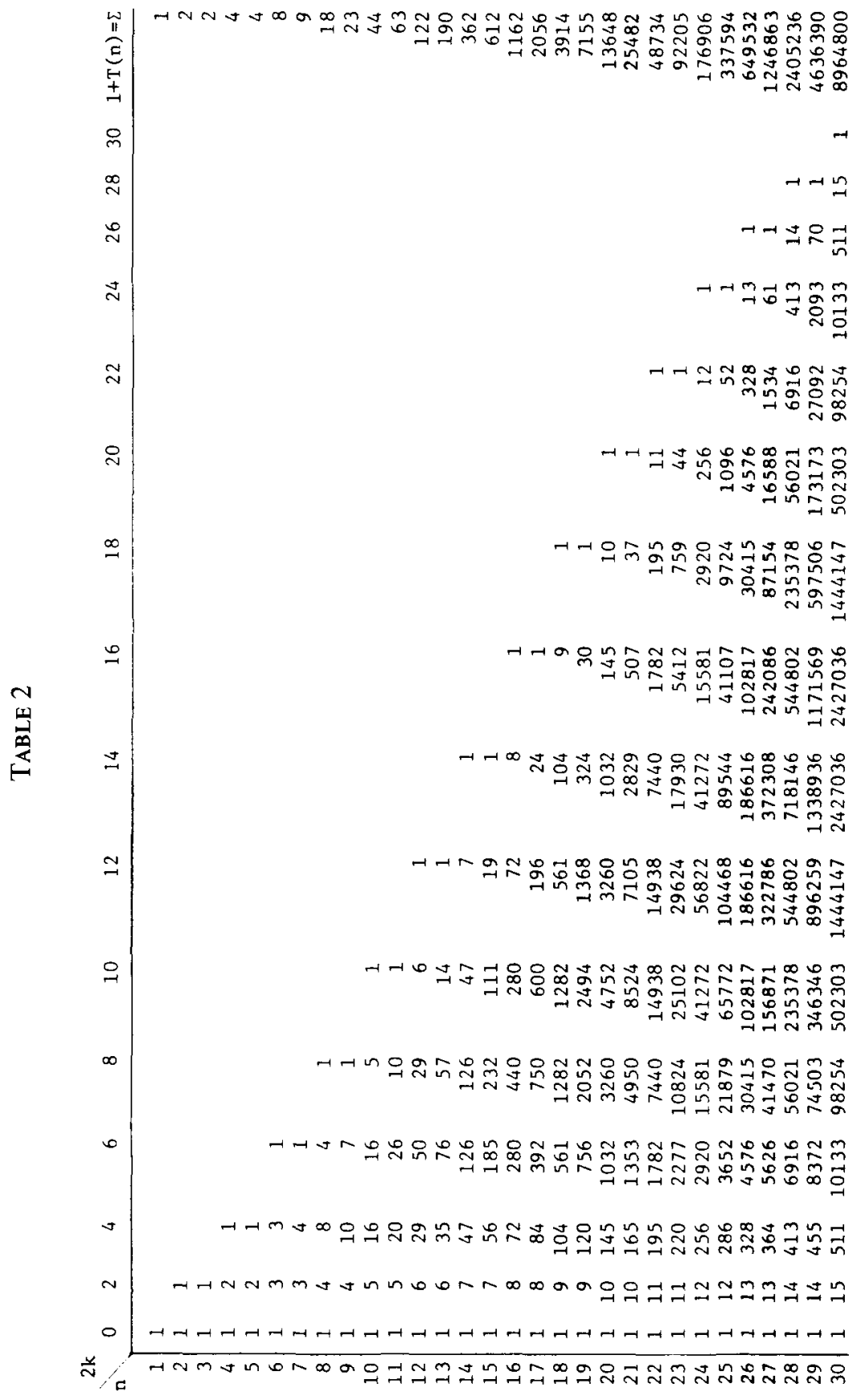


group of order $4 k$ with the symmetric group of degree two. Thus $G$ has order $g=8 k$.

(i) All $\left(\begin{array}{c}2 k \\ k\end{array}\right)$ of the sequences are left invariant by the identity of $G$; no sequence is invariant under complementation.

(ii) As in the proof of Theorem 1, it follows that a cyclic sequence $a_{0} a_{1} \ldots a_{2 k-1}$ left invariant by rotation through $s$ places must consist of $2 k / d$ repetitions of a subsequence of length $d$, where $d=\operatorname{gcd}(2 k, s)$. But, for balance, $d$ must be even; if $d=2 e$, then there are $\left(\begin{array}{c}2 e \\ e\end{array}\right)$ possible sequences.

Hence for each $d$ such that $d \mid 2 k, 1 \leqslant d<2 k$, and $d=2 e$, there are $\Phi(2 k / d)=$ $\Phi(k / e)$ rotations, each leaving $\left(\begin{array}{c}2 e \\ e\end{array}\right)$ sequences in $S$ invariant.

(iii) Similarly, a balanced cyclic sequence of period $2 k$ left invariant by rotation through $s$ places and complementation consists of $2 \mathrm{k} / 2 \mathrm{~d}$ repetitions of a sequence of length $2 d$ of the form

$$
a_{0} a_{1} \ldots a_{d-1} \overline{a_{0}} \overline{a_{1}} \ldots \overline{a_{d-1}},
$$

and there are $2^{d}$ such possible sequences.

Hence, for each $d$ such that $d \mid k$, there are $\Phi(2 k / d)$ rotations each leaving $2^{d}$ sequences in $S$ invariant.

(iv) Since the balanced cyclic sequences have even period, two types of axes of reflection are possible: $k$ axes passing between symbols $a_{j}$ and $a_{j+1}$ and again between $a_{j+k}$ and $a_{j+1+k} ; k$ axes passing through symbols $a_{j}$ and $a_{j+k}$.

If $k$ is even, then $\left(\begin{array}{c}k \\ k / 2\end{array}\right)$ balanced sequences are left invariant by each reflection. If $k$ is odd, no balanced sequences are left invariant by reflections of the first type but $2\left(\begin{array}{c}k-1 \\ (k-1) / 2\end{array}\right)$ balanced sequences are left invariant by the $k$ reflections of the second type.

(v) Each of the $k$ reflections of the first type, together with complementation, leaves $2^{k}$ subsequences invariant. No reflection of the second type, together with complementation, leaves any sequence invariant.

(vi) The total number of invariant sequences is thus

$$
\begin{array}{ll}
\left(\begin{array}{c}
2 k \\
k
\end{array}\right) & \text { from case (i); } \\
\sum_{\substack{d \mid 2 k \\
d=2 e \\
d \neq 2 k}} \Phi(k / e)\left(\begin{array}{c}
2 e \\
e
\end{array}\right) & \text { from case (ii); } \\
\sum_{d \mid k} \Phi(2 k / d) & \text { from case (iii); } \\
2 k\left(\begin{array}{l}
2\lfloor k / 2\rfloor \\
\lfloor k / 2\rfloor
\end{array}\right) & \text { from case (iv); } \\
k .2^{k} & \text { from case (v). }
\end{array}
$$


Adding terms from (i) and (ii) gives

$$
\sum_{\substack{d / 2 k \\
d=2 e}} \Phi(k / e)\left(\begin{array}{c}
2 e \\
e
\end{array}\right)
$$

since $g=8 k$, the Theorem follows.

Table 3 gives the value of $B(2 k)$ for $k \leqslant 9$, computed both from Theorem 2 and by the sieving algorithm of Section 2 .

$\begin{array}{cc} & \text { TABle } 3 \\ k & B(2 k) \\ 1 & 1 \\ 2 & 2 \\ 3 & 3 \\ 4 & 7 \\ 5 & 13 \\ 6 & 35 \\ 7 & 85 \\ 8 & 257 \\ 9 & 765\end{array}$

Table 4 gives in decimal form all of the balanced twills on 12 harnesses.

TABLE 4

Twills on 12 harnesses (balanced) in decimal form

$\begin{array}{rrrrrrr}63 & 95 & 111 & 119 & 207 & 231 & 455 \\ 175 & 183 & 187 & 189 & 215 & 219 & 311 \\ 315 & 359 & 371 & 411 & 423 & 819 & 343 \\ 347 & 349 & 363 & 365 & 427 & 603 & 619 \\ 691 & 715 & 717 & 683 & 685 & 693 & 1365\end{array}$

ObSERvations. The listing and enumeration of the extended point twills actually describes far more than the basic twills since the word given by equation (*) can be used to describe (with appropriate changes in definitions) threading (treadling) sequences when the run is not $1 \ldots n$ or $n \ldots 1$ but some set of disjoint sets of consecutive integers covering either of these sequences. As such, the formula of Corollary 2 enumerates all possible periodic helical paths followed by a particle on the surface of a torus subject to the constraint that it circulate either clockwise or anticlockwise and that, in its spiral around the torus, it may change direction through $90^{\circ}$ a finite number of times after traversing some not necessarily constant distance. 


\section{References}

[1] W. Burnside, Theory of groups of finite order (Cambridge University Press, Second edition, 1911; Dover reprint, 1955).

[2] P. Collingwood, The techniques of rug weaving (Faber and Faber, London, 1968).

[3] N. J. Fine, 'Classes of periodic sequences', Illinois J. Math. 2 (1958), 285-302.

[4] E. N. Gilbert and J. Riordan, 'Symmetry types of periodic sequences', Illinois J. Math. 5 (1961), 657-665.

[5] Branko Grünbaum and G. C. Shephard, 'Satins and twills: an introduction to the geometry of fabrics', Math. Mag. 53 (1980), 139-161.

[6] Luther Hooper, Hand loom weaving, plain and ornamental (Pitman Press, Bath. 1910: revised reprint 1960; paperback, 1979).

[7] M. E. Laughlin, More than four (Laughlin, Sacramento, 1976).

[8] G. H. Oelsner, A handbook of weaves (McMillan, 1915).

Department of Computer Science

University of Manitoba

Winnipeg, Manitoba

Canada R3T 2N2
Department of Mathematics

University of Queensland

St. Lucia, Brisbane

Australia 